NASA Technical Memorandum 83744

\title{
Time Dependent Wave Envelope Finite Difference Analysis of Sound Propagation
}

Kenneth J. Baumeister Lewis Research Center Cleveland, Ohio

Prepared for the Ninth Aeroacoustics Conference sponsored by the American Institute of Aeronautics and Astronautics Williamsburg, Virginia, October 15-17, 1984 


\title{
TIME DEPENDENT WAVE ENVELOPE FINITE DIFFERENCE ANALYSIS OF SOUND PROPAGATION
}

\author{
Kenneth J. Baumeister \\ National Aeronautics and Space Administration \\ Lewis Research Center \\ Cleveland, Ohio 44135
}

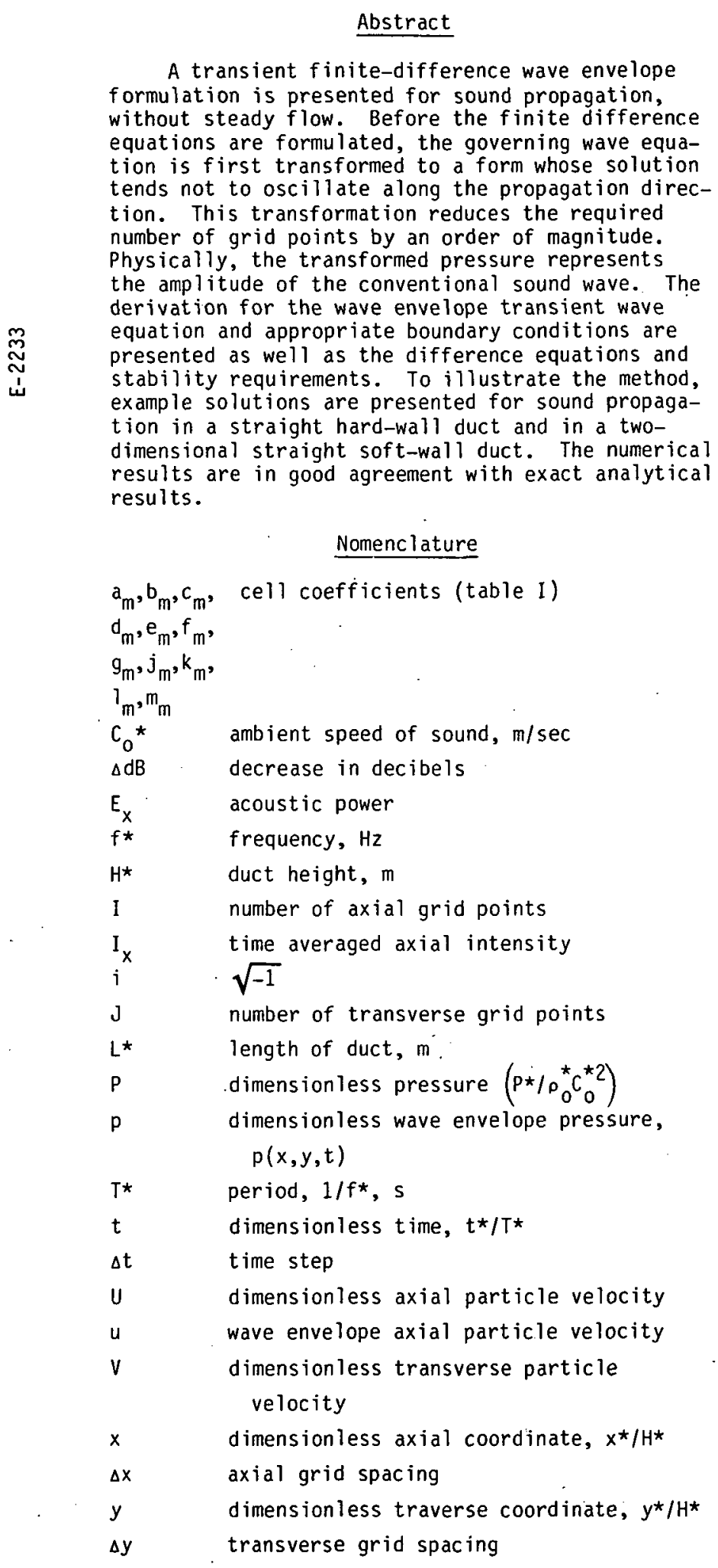

\begin{tabular}{|c|c|}
\hline$Z^{\star}$ & impedance, $\mathrm{kg} / \mathrm{m}^{2} \mathrm{~s}$ \\
\hline$\alpha$ & coefficient (Eq. (25)) \\
\hline B & coefficient (Eq. (26)) \\
\hline r & coefficient (Eq. (27)) \\
\hline$\zeta$ & specific acoustic impedance \\
\hline$n$ & dimensionless frequency (Eq. (2)) \\
\hline$n^{+}$ & wave envelope frequency \\
\hline$\lambda^{+}$ & effective wave length \\
\hline$\theta$ & dimensionless resistance \\
\hline$\rho_{0}^{*}$ & ambient air density, $\mathrm{kg} / \mathrm{m}^{3}$ \\
\hline$x$ & dimensionless reactance (for $e^{+i \omega^{\star} t *}$ ) \\
\hline$\omega^{\star}$ & angular frequency, $\mathrm{rad} / \mathrm{S}$ \\
\hline \multicolumn{2}{|c|}{ Subscripts } \\
\hline e & exit condition \\
\hline$i$ & axial grid index \\
\hline j & transverse grid index \\
\hline m & cell index \\
\hline $\max$ & maximum stable time increment (Eq. (29)) \\
\hline 0 & ambient condition \\
\hline \multicolumn{2}{|c|}{ Superscripts } \\
\hline * & dimensional qu \\
\hline$k$ & \\
\hline
\end{tabular}

Introduction

"Steady-state" finite difference and finite element theories 1,2 have been developed to study sound propagation in free space and in complex ducts with axial variations in cross-sectional area, wall liner impedance (absorbers) and with gradients in flow Mach number. In the "steady state" theory, the pressure and acoustic velocities are assumed to be simple harmonic functions of time; thus, the equations governing sqund propagation ( 1 inearized gas dynamic equations 3 (pg. 5) become independent of time. Generally the "steady state" finite difference and finite element numerical algorithms have been limited to low frequencies and short ducts, because many grid points or elements were required to resolve the axial wave length of sound and because of the large matrices associated with the solution of time independent equations.

In many practical situations something (a suppressor's impedance or a turboprop's blade geometry) needs to be optimized in some manner to obtain the maximum sound power reduction. In the optimi$z$ ation process, hundreds of calculations are often required to determine the desired configurations. Therefore, any significant reduction in the number of grid points or elements in a numerical analysis will reduce the cost of obtaining the desired optimization. 
Beginning in 1974, Baumeister ${ }^{4-6}$ developed a wave envelope concept which was used to significantly reduce (two orders of magnitude) the number of grid points associated with the "steady state" solution of high frequency sound propagation in ducts. This concept involved a transformation of the wave equation into a form whose solution does not oscillate in the axial direction. The use of the wave envelope theory drastically cut the computer costs associated with the optimization of multi-segmented liners. Nayfeh and Kaiser 8,9 extended the method for sound propagation in nonuniform ducts and with sheared flow. Astley and Eversman 10 applied the wave envelope approach very successfully in finite element duct sound transmission studies. Finally, these same authors 11 made a very significant extension of the wave envelope concept to describe simultaneously the induct propagation of sound in a turbof an nacelle and its subsequent far field radiation pattern. Consequently, numerical techniques can now be employed to study sound propagation in both the internal and far field regions of a duct provided the sound frequency is reasonably low.

\& In order to further reduce computer storage and run times, as an alternate to the "steadystate" theories, time dependent numerical solutions were developed by Baumeister ${ }^{12-14}$ for harmonic sound propagation in ducts. The transient formulation generally uses a time marching solution to the wave equation; consequently, the matrix storage requirements inherent in the "steady state" formulation are completely eliminated in the time dependent analysis. Only the solution vectors for pressures and velocities need be stored. By eliminating the large matrix storage requirements, numerical calculations for higher frequency sound are now possible.

The time dependent theory has also been applied to forms of the inhomogeneous wave equation by Maestrello, Bayliss and Turke 15 and Baumeister 16 . White ${ }^{17}$ has extended the transient theory by means of a mapping to variable area ducts. The simultaneous calculation of induct and the far field associated with a turbof an engine and an unflanged cylindrical duct has been calculated by White ${ }^{18}$ and Hariharan and Bayliss ${ }^{19}$, respectively.

Considering the grid point reduction of the previously discussed wave envelope theory and the elimination of matrix storage by the transient solution formulation, a logical extension would be to combine both theories. In principal, the computer storage requirements could now be reduced many orders of magnitude over previous theories making possible calculations with higher frequencies of three-dimensional fields.

To combine the theories, the wave envelope transient wave equation and appropriate boundary conditions will first be derived. The theories will be presented for a two-dimensional soft-wall duct without mean flow. Next, the complete set of difference equations and stability requirements will. be presented. Finally, sample calculations are presented for plane wave propagation in a hardwalled straight duct and for the attenuation provided by a two-dimensional soft-walled duct.

\section{Grid Point Problem}

The propagation of sound in a duct is described by the wave equation and appropriate source and impedance boundary conditions. The wave equation in a two-dimensional rectangular duct can be expressed in dimensionless form as:

$$
\frac{1}{c^{2}} \frac{\partial^{2} p}{\partial t^{2}}=\frac{\partial^{2} p}{\partial x^{2}}+\frac{\partial^{2} p}{\partial y^{2}}
$$

The "steady state" version of this wave equation assumes $P$ is proportional to $e^{i \omega t}$ such that the left-hand side is replaced by $(i \omega / c)^{2} \mathrm{p}$. Therefore, as mentioned in the introduction, the "steady

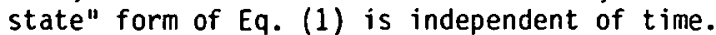

The usual notation for pressure, distance coordinates and speed of sound are used. These and other symbols are defined in the nomenclature. Here, the dimensionless speed of sound $C$ and the dimensionless frequency $\eta$ are defined as

$$
C=\frac{1}{n}=\frac{C^{\star}}{H^{\star} f^{\star}}
$$

The asterisks denote dimensional quantities.

In a finite difference numerical analysis of the wave equation, the continuous acoustic field is lumped into a series of grid points (Fig. 1). Next, the wave equation is expressed in difference form. The difference equations are then solved by a time marching process to obtain the pressure at each grid point. Obviously, the more grid points required in a solution the greater the computer storage and run times.

Consider a hard-wall duct, infinite in extent with a plane pressure wave şinusoidal in time at $x=0\left(P(0, t)=e^{i \omega t}=e^{i 2 \pi t}\right)$. The solution of $\mathrm{Eq} .1$ for the pressure yields $12^{\circ}, \mathrm{Eq} .27$ is

$$
\frac{P(x, t)}{e^{i 2 \pi t}}=e^{-i 2 \pi n x}
$$

The finite-difference approximation for the "steady state" acoustic pressure in a semi-infinite hardwall duct 12 and the analytical solution (Eq. (3)) are presented in Fig. 2 for $x$ greater than 0 and less than 1. By a series of numerical experiments, the number of axial grid points, I, necessary to obtain pressure profiles, velocity and intensities accurate to about 4 percent were determined to be

$$
I=12 n\left(L^{\star} / H^{\star}\right)+1
$$

Thus, for the case of unit frequency $(n=1)$ and unit duct length to height ratio $\left(L^{*} / H^{*}=1\right)$ shown in Fig. 2, 13 grid points were necessary to describe adequately the sinusoidal form of the spa$t i a l$ pressure dependence. If the frequency or length is doubled, clearly twice as many points will be required to describe the wave, since two wave lengths of sound must be resolved. The total grid points will be the product of $I$ and the number of grid points $\mathrm{J}$ in the transverse direction.

Next, a concept is considered which eliminates the direct dependency of the number of axial grid points on $\eta$ and $\left[{ }^{\star} / H^{\star}\right.$. 


\section{Wave Envelope Equation}

Consider the example case of a soft-wall duct with an $L^{\star} / H^{\star}$ of 3 and an inlet plane wave with a dimensionless frequency $n$ equal to 1 . A typical pressure (real part) profile in a suppressor duct is shown in Fig. 3 by the heavy solid line, while the dashed lines represent the envelope of the solid pressure wave amplitude. The pressure amplitude decreases in the axial direction down the length of the duct because of acoustic energy dissipation at the suppressor wall. However, the basic axial variation of the pressure is similar to that in a hard-wall duct. Thus, the number of grid points (open circles in Fig. 3) still depends on frequency $n$ and the dimensionless duct length $L * / H *$ (Eq. (4)).

From Eq. (4), the number of grid points I needed would be about 37 . However, if the basic wave equation could be transformed so that it would describe the envelope (dashed lines) of the pressure rather than the pressure wave itself, the 37 point requirement could be greatly reduced. As shown in Fig. 3, five points are used to describe the pressure envelope.

The assumption is now made that the pressure $P(x, y, t)$ can be separated into the following

$$
P(x, y, t)=p(x, y, t) e^{-i 2 \pi n^{+} x}
$$

where $p(x, y, t)$ represents the envelope of the pressure wave as shown by the dashed line in Fig. 3 and where

$$
\eta^{+}=H^{*} / \lambda^{*}
$$

with $\lambda^{*}$ representing the effective axial wavelength of the pressure in the duct. Substituting Eq. (5) into the wave Eq. (1) yields a new time dependent governing differential equation called the time dependent wave envelope equation:

$\frac{\partial^{2} p}{\partial t^{2}}=\frac{1}{\eta^{2}}\left(\frac{\partial^{2} p}{\partial x^{2}}+\frac{\partial^{2} p}{\partial y^{2}}\right)-\frac{i 4 \pi \eta}{\eta^{2}} \frac{\partial p}{\partial x}-(2 \pi)^{2}\left(\frac{\eta^{+}}{\eta}\right)^{2} p$

In free filed applications, the axial wave length $\lambda^{*}$ is known. Thus, the use of the wave envelope concept is relatively straightforward. However, in soft-wall ducts where multi-modal propagation occurs, the axial wave length $\lambda^{*}$ is not known precisely; therefore, the problem of picking $n^{+}$to exactly define the wave length must be considered. In the "steady-state" wave envelope solution presented ${ }^{5}, \lambda^{\star}$ in the soft-wall duct was assumed equal to $\lambda^{*}$ for a plane wave in a hardwall duct. This assumption was found to yield excellent results in the "steady-state" wave length solution. Therefore, the same assumption will be used here in the solution of Eq. (7).

As shown in Ref. 5 (Fig. 4), it is necessary only to pick a value of $\lambda^{*}$ or $\left(\pi^{+}\right)$in the vicinity of the average wave length to get large savings in the grid points required for a finitedifference analysis. Therefore, for a plane wave source as considered herein, $n^{+}$will be assumed to equal $n$. In a problem where the source might be some higher order mode, $n^{+}$would be assumed to be a value associated with that mode.

\section{Governing Equations and Boundary Conditions}

Besides the wave envelope Eq. (7), the equations for the acoustic velocity, soft-wall boundary conditions, and acoustic intensity are required to obtain an expression for the attenuation of a softwall duct. These equations are now presented.

\section{Linearized Momentum Equation}

In the absence of mean flow, the $x$ and $y$ dimensionless momentum equations can be written as

$$
\begin{aligned}
& \frac{\partial U}{\partial t}=-\frac{1}{n} \frac{\partial P}{\partial x} \\
& \frac{\partial V}{\partial t}=-\frac{1}{n} \frac{\partial P}{\partial y}
\end{aligned}
$$

or in terms of the wave envelope parameters

$$
\begin{gathered}
\frac{\partial u}{\partial t}=-\frac{1}{\eta}\left[\frac{\partial p}{\partial x}-i 2 \pi \eta+p\right] \\
\frac{\partial v}{\partial t}=-\frac{1}{n} \frac{\partial p}{\partial y}
\end{gathered}
$$

Wall Boundary Condition

In the transverse direction, the acoustic impedance at the wall shown in Fig. 1 is defined as the ratio of pressure to the transverse velocity

$$
\zeta=\frac{Z^{\star}}{\rho_{0}^{\star} C_{0}^{\star}}=\frac{P}{V}
$$

Substituting Eq. (12) into Eq. (9) to eliminate V yields

$$
\frac{\partial P}{\partial y}=-\frac{n}{\zeta} \frac{\partial P}{\partial t}
$$

or in terms of the wave envelope parameters

$$
\frac{\partial p}{\partial y}=-\frac{n}{\zeta} \frac{\partial p}{\partial t}
$$

Equation (14) sets the pressure gradient along the upper and lower walls.

\section{Exit Impedance}

In a manner similar to the wall impedance, the axial impedance at the duct exit can be defined as

$$
\zeta_{e}=\frac{P\left(L^{\star} / H^{\star}, y, t\right)}{U\left(L^{\star} / H^{\star}, y, t\right)}
$$

Again, substituting Eq. (15) into Eq. (8) yields

$$
\frac{\partial P}{\partial x}=-\frac{\eta}{\zeta_{e}} \frac{\partial P}{\partial t}
$$

or substituting Eq. (5) into Eq. (16) yields

$$
\frac{\partial p}{\partial x}=-\frac{\eta}{\zeta_{e}} \frac{\partial p}{\partial t}+i 2 \pi \eta^{+} p
$$

For the plane wave propagation to be considered herein, $\zeta_{e}$ is taken as 1 , which is exact for plane 
wave propagation in an infinite hard-wall duct. Also choosing $5 \mathrm{e}$ to be 1 has lead to close agreement between numerical and analytical results for plane wave propagation into a soft-wall duct 4 .

\section{Entrance Condition}

The boundary condition at the source plane $P(0, y, t)$ allows for transverse variations in pressure; however, as mentioned earlier, the numerical technique will be compared later to previous solutions in which the pressure and acoustic velocities were assumed to be plane waves at the entrance and to vary as $e^{i 2 \pi t}$. Therefore, the source boundary condition used here is

$$
\dot{P}(0, y, t)=p(0, y, t)=e^{i 2 \pi t} t>0
$$

\section{Initial Condition}

For times equal or less than zero, the duct is assumed quiescent, that is, the acoustic pressure and velocities are taken to be zero. For times greater than zero, the application of the noise source (e.g., Eq. (18)) will drive the pressures in the duct.

\section{Acoustic Intensity}

The sound power that leaves a duct and reaches the far-field is related to the time-averaged acoustic intensity, defined as

$$
I_{x}=1 / 2 R E\left\{\left(\overline{\left(P / e^{i 2 \pi t}\right.}\right) \times\left(U / e^{i 2 \pi t}\right)\right\}
$$

where the bar represents the complex conjugate.

The total dimensionless acoustic power is the integral of the intensity across the test section

$$
E x=\int_{0}^{1} I_{x}(x, y) d y
$$

By definition, the sound attenuation can be written as

$$
\Delta d B=10 \log _{10}(E x / E 0)
$$

Next, the difference form of these equations will be presented.

\section{Difference Equations}

Instead of a continuous solution for pressure in space and time, the finite-difference approximations will determine the pressure at isolated grid points in space as shown in Fig. 1 and at discrete time steps $\Delta t$. Starting from the known initial conditions at $t=0$ and the boundary conditions, the finite difference algorithm will march out the solution to later times. The development for the algorithm that applies to each cell in Fig. 1 will now be discussed.

\section{Central Region (Cell 1)}

Away from the duct boundaries, in cell 1 of Fig. 1, the first and second derivatives in the wave envelope equation (e.g., Eq. (7)) can be represented by the usual central differences in time and space 20,21

$$
\begin{aligned}
\left(p_{i, j}^{k+1}-\right. & \left.2 p_{i, j}^{k}+p_{i, j}^{k-1}\right) / \Delta t^{2} \\
& =\left(\frac{1}{\eta^{2}}\right)\left[\left(p_{i+1, j}^{k}-2 p_{i, j}^{k}+p_{i-1,1}^{k}\right) / \Delta x^{2}\right] \\
& +\frac{1}{n^{2}}\left[\left(p_{i, j+1}^{k}-2 p_{i, j}^{k}+p_{i, j-1}^{k}\right) / \Delta y^{2}\right] \\
& -i 4 \pi \frac{\eta^{+}}{n^{2}}\left[\left(p_{i+1, j}^{k}-p_{i-1, j}^{k}\right) / 2 \Delta x\right] \\
& -\left(2 \pi \eta^{+} / \eta\right)^{2} p_{i, j}^{k}
\end{aligned}
$$

where $\boldsymbol{i}$ and $\mathbf{j}$ denote the space indices, $\dot{k}$ the time index, and $\Delta x, \Delta y$ and $\Delta t$ are the space and time mesh spacing, respectively. All spacings are assumed constant. The time is defined as

$$
t^{k+1}=t^{k}+\Delta t
$$

Solving Eq. (24) for $p_{i, j}^{k+1}$ yields

$$
\begin{aligned}
& p_{i, j}^{k+1} \\
& =\alpha\left\{\left[\left(\frac{\Delta y}{\Delta x}\right)^{2}+\frac{i \beta}{2 \Delta x \alpha}\right] p_{i-1, j}^{k}\right. \\
& +p_{i, j-1}^{k}-2\left[1+\left(\frac{\Delta y}{\Delta x}\right)^{2}-\frac{1}{\alpha}+\frac{\gamma}{2 \alpha}\right] p_{i, j}^{k} \\
& \left.+p_{i, j+1}^{k}+\left[\left(\frac{\Delta y}{\Delta x}\right)^{2}-\frac{i \beta}{2 \Delta x \alpha}\right] p_{i+1, j}^{k}\right\}-p_{i, j}^{k-1}
\end{aligned}
$$

$$
\begin{aligned}
& \alpha=\Delta t^{2} / n^{2} \Delta y^{2} \\
& \beta=4 \pi n^{+} \Delta t^{2} / \eta^{2} \\
& \gamma=\left(2 \pi \Delta t n^{+} / n\right)^{2}
\end{aligned}
$$

Equation (24) is an algorithm which permits marching-out solutions from known values of pressure at times associated with $k$ and $k-1$. The procedure is explicit since all the past values of $p k$ are known as the new values of $k+1$ are computed. For the special case at $t=0$, the values of the pressure associated with the $k-1$ value are zero from the assumed initial condition.

\section{Boundary Condition (Cells 2 to 6 )}

The expression for the difference equations at the wall boundaries are complicated by the impedance conditions and the change in geometry of the cells 2 to 6 in Fig. 1. The governing difference equations can be developed by an integration process in which the wave envelope equation (e.g., Eq. (9)) is integrated over the area of the cells and time. The procedure for the temporal and 
spatial integration over the cell area is fully documented 6,12 .

For ease in bookkeeping the solution for $P_{j}^{k}$ for the various cells is written in the général form

$$
\begin{aligned}
& p_{i, j}^{k+1}=\frac{a}{\left[1-a f_{m}\right.}\left[\left(a_{m}-\frac{i \beta k_{m}}{a}\right) p_{i-1, j}^{k}\right. \\
& +b_{m} p_{i, j-1}^{k}+\left(c_{m}+\frac{2}{a}-\frac{i \beta \ell_{m}}{\alpha}-\frac{\gamma}{\alpha}+i j_{m}\right) p_{i, j}^{k} \\
& \left.+d_{m} p_{i, j+1}^{k}+\left(e_{m}-\frac{i \beta m_{m}}{\alpha}\right) p_{i+1, j}^{k}\right] \\
& -\frac{\left[1-\alpha g_{m}\right]}{\left[1-\alpha f_{m}\right]} p_{i, j}^{k-1}
\end{aligned}
$$

where the values of the coefficients $a_{m}, b_{m}$, etc. for the various cells are listed in Table I. In particular, substituting the values of the coefficients for cell 1 in Table I into Eq. (28) will reproduce Eq. (24).

\section{Stability}

In the explicit time marching approach used here, round-off errors can grow in an unbounded fashion and destroy the solution if the time increment $\Delta t$ is taken too large. The von Neumann method is often used to study the stability of the difference approximations to the wave equation. Application of the von Neumann method 21 (pg. 104) to Eq. (22) requires that the time increment be of the form

$\Delta t \leq\left(\frac{n^{2} \Delta y^{2}}{1+\left(\frac{\Delta y}{\Delta x}\right)^{2}+\frac{\pi \Delta y^{2}}{\Delta x} \eta^{+}+\left(\pi \Delta y \eta^{+}\right)^{2}}\right)^{1 / 2}$

for $n^{+}$small, Eq. (29) reduces to the conventional CFL (Courant, Friedrichs and Levy) condi$t$ ion. Because of possible effects of boundary conditions, some numerical experimentation was used to check the validity of Eq. (29).

\section{"Steady State"}

Recall, at the start of the numerical calculation, the acoustic pressures and velocities were assumed zero throughout the duct and a pressure source begins a harmonic oscillation at $x=0$ for $t>0$. The transient numerical calculation must be started and continued in time until the initial acoustic transient has died qut. This subject has been treated extensively 12,22 . For plane wave propagation, as shown in these references, the transient solution for pressure equals the "steadystate" Fourier transform solution when

$$
t>n\left(L * / H^{*}\right)
$$

\section{Axial Velocity and Intensity}

The axial velocity (Eq. (10)), acoustic intensity (Eq. (19)), and the sound attenuation (Eq. (21) can be quite easily expressed in difference form ${ }^{4-6}$. In these cases, the pressure and velocity are now assumed to be at "steady state" condition and are now functions of $e^{i 2 \pi t}$. Thus, Eq. (10) becomes

$$
u=\frac{i}{2 \pi n} \frac{\partial p}{\partial x}+\frac{n^{+}}{n} p
$$

or

$$
u_{i, j}^{k}=\frac{i}{2 \pi n}\left(\frac{p_{i+1, j}^{k}-p_{j-1, j}^{k}}{2 \Delta x}\right)+\frac{n^{+}}{n} p_{i, j}^{k}
$$

The intensity, (Eq. (19)), can be written as

$$
I_{i, j}^{k}=\frac{1}{2} \operatorname{Re}\left\{\vec{p}_{i, j}^{k} / e^{i 2 \pi t} \times u_{i, j}^{k} / e^{i 2 \pi t}\right\}
$$

where the bar over $p$ represents the complex conjugate.

Next, the total dimensionless power is determined according to Eq. (20)

$$
E_{i}^{k}=\left(\frac{1}{2} I_{i, 1}^{k}+\sum_{j=2}^{\text {LAST-1 }} I_{i, j}^{k}+I_{i, \text { LAST }}^{k}\right) \Delta y
$$

and finally the attenuation is

$$
\begin{aligned}
& \Delta d B=10 \log _{10}\left(\frac{E_{i}^{k}}{E_{1}^{k}}\right) \\
& \text { Sample Calculations }
\end{aligned}
$$

In two sample problems to follow, the timedependent wave envelope results will be compared to closed form analytical solutions. First, the simple case of plane waves propagating down a hardwall one-dimensional duct is presented. This case allows comparison of the numerical and analytical pressure profiles down the length of the duct. The second example compares the numerical and analytical predictions of the attenuation in a soft-wall two-dimensional duct.

\section{One-Dimensional Hard-Wall Duct}

Numerical and analytical values of the pressure were computed for the special case of a hardwall duct with a $L^{*} / H^{*}$ of $1, \eta$ of 1 and an inlet plane wave. The analytical value from Eq. (5) yields

$$
\frac{p(x, y, t)}{e^{-i 2 \pi t}}=1+i 0.0
$$

As seen in Fig. 4, the agreement between the numerical and analytical resuits is reasonable. A comparison with Fig. 2 indicates the essential differences between the transformed numerical solution and the conventional numerical solution for the same problem.

\section{Two-Dimensional Soft-Wal.l Duct}

As another example of the transient wave envelope formulation, the noise attenuation at the optimum point (point of maximum attenuation in the impedance plane) is now calculated for a twodimensional duct with $L^{*} / H^{*}$ values varying 
between 0.5 and 6 and input plane waves with dimensionless frequencies $n$ of 1,2 , and 5 . This range of dimensionless parameters essentially covers the practical range of application to turbojet exhaust suppressors.

For a plane entrance pressure profile the closed form analytical results show that the values of specific acoustic wall impedance $\zeta$ associated with the optimum impedance used in the numerical analysis were getermined from the analytical techniques 23,24 and are listed in Table II.

The numerically calculated attenuations are compare to $_{\text {the }}$ corresponding analytical results 23,24 which are applicable to inf inite ducts. The numerical results (symbols) and the analytical results (1ines) for the maximum attenuation are shown in Fig. 5. The analytical and numerical results are in very good agreement. A slight deviation occurred at the very high attenuation associated with the low frequency case $n=1$ at $L^{\star} / H^{\star}$ of 4 . For the low frequency case, it is suggested that more points be employed.

Based on the wave envelope concept, the numerical calculations shown in Fig. 5 used 30 grid points in the axial direction. For $n=5$ and $L^{\star} / H^{\star}=6$, the standard finite difference technique required 360 grid points, according to Eq. (5). Thus, for a $J=10$, the total number of grid points has been reduced from 3600 to 300 when the wave envelope concept is employed. This represents an order of magnitude savings in computer storage and computational time.

\section{Concluding Remarks}

Transient finite difference solutions using the wave envelope concept are presented for plane wave sound propagation in a one-dimensional hardwall duct and a two-dimensional soft-wall duct for zero Mach number. The results show the numerical procedure to be in agreement with the corresponding exact analytical results.

The wave envelope approach to the numerical problem reduces the number of grid points in the difference solution by an order of magnitude compared to the conventional difference technique. Table III shows the large reduction in computer storage requirements by employing both the transient and wave envelope techniques as compared to the conventional "steady-state" theory. Clearly, numerical solutions for acoustic propagation in complex ducts can now be determined with reasonable storage and computational times.

\section{References}

1. Baumeister, K. J., "Numerical Techniques in Linear Duct Acoustics - A Status Report," ASME Paper 80-WA/NC-2, Nov. 1980.

2. Baumeister, K. J., "Numerical Techniques in Linear Duct Acoustics, 1980-81 Update," NASA TM-82730, 1981 .

3. Goldstein, M. E., Aeroacoustics, McGraw-Hill, New York, 1976.

4. Baumeister, K. J., "Analys is of Sound Propagation in Ducts Using the Wave Envelope Concept," NASA TN D-7719, 1974.
5. Baumeister, K. J., "Wave Envelope of Sound Propagation in Ducts with Variable Axial Impedance." Aeroacoustics: Fan Noise and Control; Duct Acoustics; Rotor Noise, Progress in Astronautics and Aeronautics Series, Vol. 44, AIAA, edited by I. R. Schwartz, H. T. Nagamatsu, and W. Strahle, New York, 1976, pp. 451-474.

6. Baumeister, K. J., "Finite-Difference Theory for Sound Propagation in a Lined Duct with Uniform Flow Using the Wave Envelope Concept," NASA TP $1001,1977$.

7. Baumeister, K. J., "Evaluation of Optimized Multisectioned Acoustic Liners," AIAA Journal, Vol. 17, Nov. 1979, pp. 1185-1192.

8. Kaiser, J. E., and Nayfeh, A. H., "A WaveEnvelope Technique for Wave Propagation in Non-uniform Ductś," AIAA Journal, Vol. 15, Apr. 1977, pp. 533-537.

9: Nayfeh, A. H., Shaker, B. S., and Kaiser, J. E., "Transmission of Sound Through Nonuniform Circular Ducts with Compressible Mean Flows," AIAA Journal, Vol. 18, May 1980, pp. 515-525.

10. Astley, R. J., and Eversman, W., "A Note on the Utility of a Wave Envelope Approach in Finite Element Duct Transmission Studies," Journal of Sound and Vibration, Vol. 76, June 22, 1981, pp. 595-601.

11. Astley, R. J., and Eversman, W., "Wave Envelope and Infinite Element Schemes for Fan Noise Radiation from Turbofan Inlets," AIAA Paper 83-0709, Apr. 1983.

12. Baumeister, K. J., "Time-Dependent Difference Theory for Noise Propagation in a TwoDimensional Duct," AIAA Journal, Vol. 18, Dec. 1980, pp. 1470-1476.

13. Baumeister, K. J., "A Time Dependent Difference Theory for Sound Propagation in Ducts with Flow," 98th Meeting of the Acoustical Society of America, Salt Lake City, UT, Nov. 26-30, 1979.

14. Baumeister, K. J., "Time Dependent Difference Theory for Sound Propagation in Axisymmetric Ducts with Plug Flow," AIAA Paper 80-1017, June 1980 .

15. Maestrello, L., Bayliss, A., and Turkel, E., "On the Interaction of a Sound Pulse with the Shear Layer of an Axisymmetric Jet, "Journal of Sound and Vibration, Vol. 74, Jan. 22, 1981, pp. 281-301.

16. Baumeister, K. J., "Transient Difference Solutions of the Inhomogeneous Wave EquationSimulation of the Green's Function, "AIAA Paper 83-0667, Apr. 1983.

17. White, J. W., "A General Mapping Procedure for Variable Area Duct Acoustics," AIAA Journal, Vol. 20, July 1982, pp. 880-884.

18. White, J. W., and Raad, P. E., "A Mapped Factored Implicit Scheme For the Computation of Duct and Far Field Acoustics," AIAA Paper 84-0501, Jan. 1984. 
19. Hartharan, S. L., and Bayliss, A., "Radiation of Sound From Unflanged Cylindrical Ducts," NASA CR-172171, 1983.

20. Gerald, C. F., Applied Numerical Analysis, 2nd ed., Addision-Westey, Reading, Mass., 1978.

21. Clark, M., and Hansen, K. F., Numerical Methods of Reactor Analysis, Academic Press, New York, 1964.
22. Pearson, J. D., "The Transient Motion of Sound Waves in Tubes." Quarterly Journal of Mechanics and Applied Mathematics, Vol. 6, Pt. 3, 1953, pp. 313-335.

23. Rice, Edward J., "Attenuation of Sound in Soft-walled Circular Ducts," Aerodynamic Noise, Proceedings of the AFOSR-UT/AS Symposium, edited by H. S. Ribner, University of Toronto, Toronto, 1968, pp. 229-250.

24. Rice, E. J., "Performance of Noise Suppressors for a Full-Scale Fan for Turbofan Engines." AIAA Paper 71-183, Jan. 1971. 
TABLE I. - COEFFICIENTS IN DIFFERENCE EQUATIONS

\begin{tabular}{|c|c|c|c|c|c|c|c|c|c|c|c|c|}
\hline \multirow{2}{*}{$\begin{array}{c}\text { Cell } \\
\text { index, } \\
\text { m }\end{array}$} & \multirow[b]{2}{*}{$a_{m}$} & \multirow[b]{2}{*}{$b_{m}$} & \multicolumn{10}{|c|}{ Difference elements ${ }^{a}$} \\
\hline & & & & $c_{m}$ & $d_{m}$ & $e_{m}$ & $f_{m}$ & $9 m$ & $j_{m}$ & $k_{m}$ & $1_{m}$ & $m^{m}$ \\
\hline 1 & $\left(\frac{\Delta y}{\Delta x}\right)^{2}$ & 1 & -2 & {$\left[1+\left(\frac{\Delta y}{\Delta x}\right)^{2}\right.$} & 1 & $\left(\frac{\Delta y}{\Delta x}\right)^{2}$ & 0 & 0 & 0 & $-\frac{1}{2 \Delta x}$ & $\underline{0}$ & $\frac{1}{2 \Delta x}$ \\
\hline 2 & $a_{1}$ & 2 & & $c_{1}$ & 0 & $e_{1}$ & $-\frac{n}{\zeta} \frac{\Delta y}{\Delta t}$ & $\frac{\eta}{\zeta} \frac{\Delta y}{\Delta t}$ & 0 & $k_{1}$ & 0 & $m_{1}$ \\
\hline 3 & $a_{1}$ & 0 & & $c_{1}$ & 2 & $e_{1}$ & $-\frac{n}{\zeta} \frac{\Delta y}{\Delta t}$ & $\frac{\eta}{\zeta} \frac{\Delta y}{\Delta t}$ & 0 & $k_{1}$ & 0 & $\mathrm{~m}_{1}$ \\
\hline 4 & $2 a_{1}$ & 1 & & $c_{1}$ & 1 & 0 & $-\frac{n \Delta y^{2}}{5 e^{\Delta t \Delta x}}$ & $\frac{n \Delta y^{2}}{\zeta e^{\Delta t \Delta x}}$ & $\frac{4 \pi \pi^{+} \Delta y^{2}}{\Delta x}$ & $-\frac{1}{\Delta x}$ & $\frac{1}{\Delta x}$ & 0 \\
\hline 5 & $2 a_{1}$ & 2 & & $c_{1}$ & 0 & 0 & $f_{2}+f_{4}$ & $g_{2}+g_{4}$ & $j_{4}$ & $k_{4}$ & $1_{4}$ & 0 \\
\hline 6 & $2 a_{1}$ & 0 & & $c_{1}$ & 2 & 0 & $f_{3}+f_{4}$ & $g_{3}+g_{4}$ & $\mathrm{j}_{4}$ & $k_{4}$ & $1_{4}$ & 0 \\
\hline
\end{tabular}

TABLE II. - OPTIMUM IMPEDANCE

AND ATTENUATION FOR UNIFORM

RECTANGULAR INFINITE DUCT

WITH PLANE WAVE INPUT,

$\zeta=\theta+i x$

\begin{tabular}{|l|l|l|l|c|}
\hline$\eta$ & $L^{\star} / H^{\star}$ & $\sigma$ & $X$ & $\begin{array}{c}\text { Analytical } \\
\text { attenuation, } \\
-\Delta \mathrm{dB}\end{array}$ \\
\hline 1 & 0.5 & 0.23 & -0.55 & 4.0 \\
& 1 & .46 & -.92 & 8.2 \\
& 2 & .78 & -1.05 & 22.6 \\
& 3 & .9 & -.93 & 39.9 \\
& 4 & .92 & -.85 & 56 \\
\hline 2 & .5 & .34 & -.86 & 2.2 \\
& 1 & .47 & -1.32 & 3.2 \\
& 2 & .86 & -2.0 & 6.7 \\
& 3 & 1.32 & -2.35 & 12.0 \\
& 4 & 1.71 & -2.33 & 18.8 \\
& 6 & 2.0 & -1.9 & 34.8 \\
\hline 5 & .5 & .6 & -1.53 & 1.1 \\
& 1 & .84 & -2.2 & 1.7 \\
& 2 & 1.28 & -3.5 & 2.5 \\
& 3 & 1.35 & -3.87 & 3.4 \\
& 4 & 1.72 & -4.3 & 5.0 \\
& 5 & 2.12 & -5.1 & 6.3 \\
& 6 & 2.55 & -5.5 & 7.9 \\
\hline
\end{tabular}


TABLE III. - GRID POINT AND STORAGE REQUIREMENTS (REAL AND IMAGINARY) FOR $n=6, L \star / H^{\star}=5, \mathrm{~J}=10$

\begin{tabular}{|l|c|c|c|c|}
\hline \multicolumn{1}{|c|}{ Method } & $\begin{array}{c}\text { Grid } \\
\text { points }\end{array}$ & Matrix & $\begin{array}{c}\text { Solution } \\
\text { vector }\end{array}$ & $\begin{array}{c}\text { Total } \\
\text { storage }\end{array}$ \\
\hline Steady state & 3600 & $26 \times 10^{6}$ & 7200 & $26 \times 10^{6}$ \\
Steady state wave & 100 & 20,000 & 200 & 20,200 \\
envelopel & & 0 & 7220 & 14,440 \\
Transient 12 & 3610 & 0 & 620 & 1,240 \\
\hline Transient wave envelope & 310 & 0 &. \\
\hline
\end{tabular}




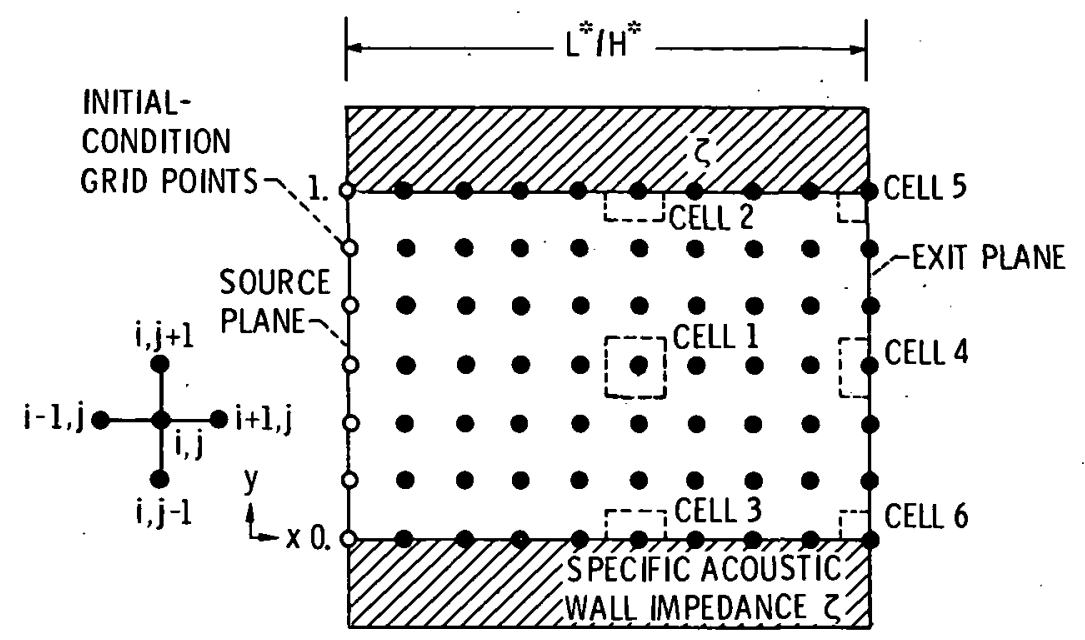

Figure 1. - Grid-point representation of two dimentional duct.

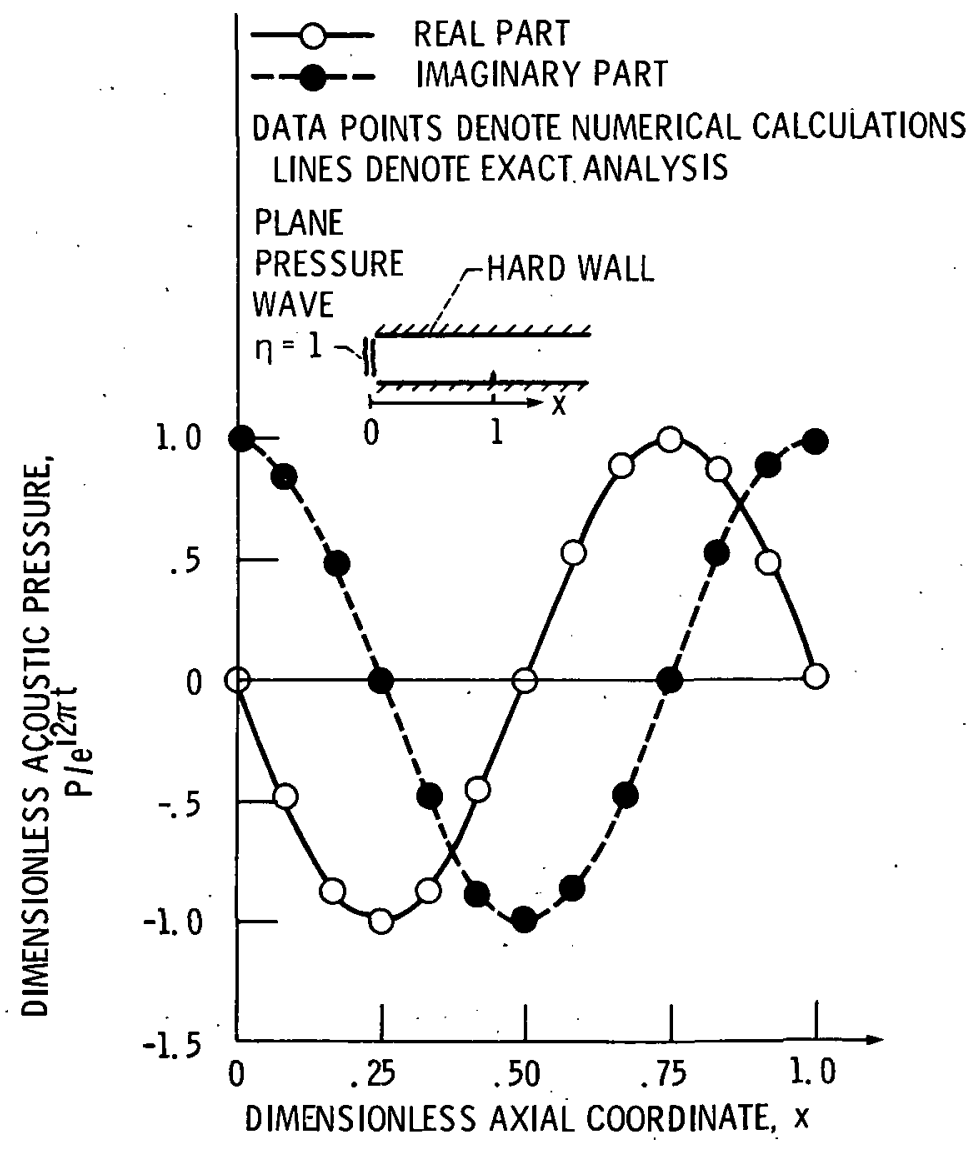

Figure 2. - Analytical and numerical pressure profiles for one dimensional sound propagation in hard-wall duct, $\eta=1$. 


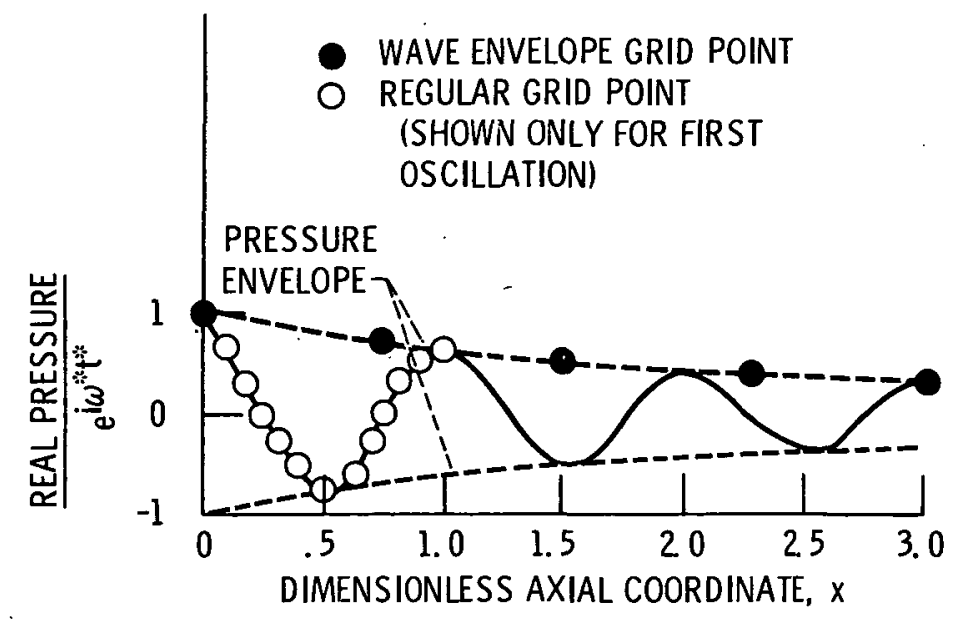

Flgure 3. - Typical pressure profile for sound propagation in a soft-wall duct for dimensionless frequency $\eta=1$ and duct length $L^{*} / H^{*}=3$.

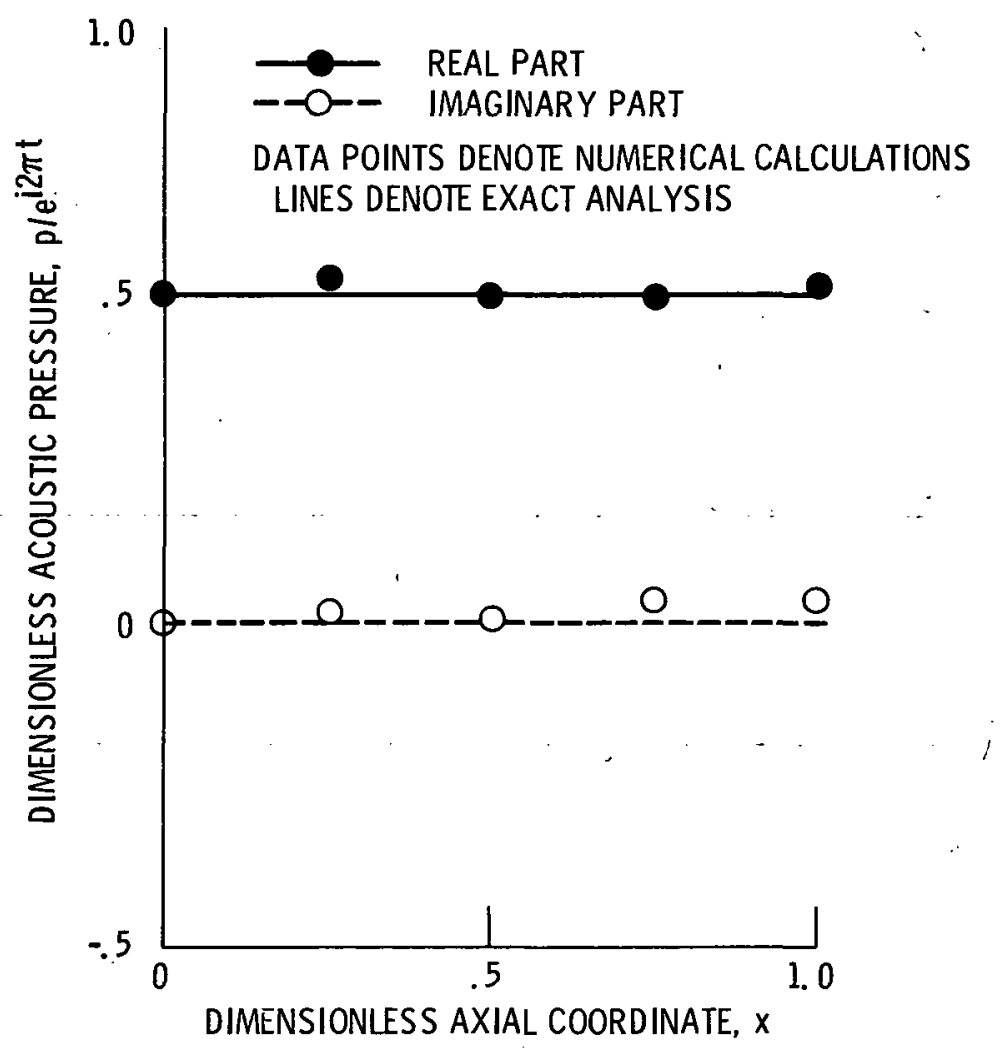

Figure 4. - Analytical and numerical dimensionless acoustic pressure profiles for sound propagating in a hard wall duct. $\quad(n=1$, $L^{*} / H^{*}=1, I=5, J=10, t=5.0, \Delta t=$ $0.5 \times \Delta \mathrm{t}_{\text {max }}$ ). 


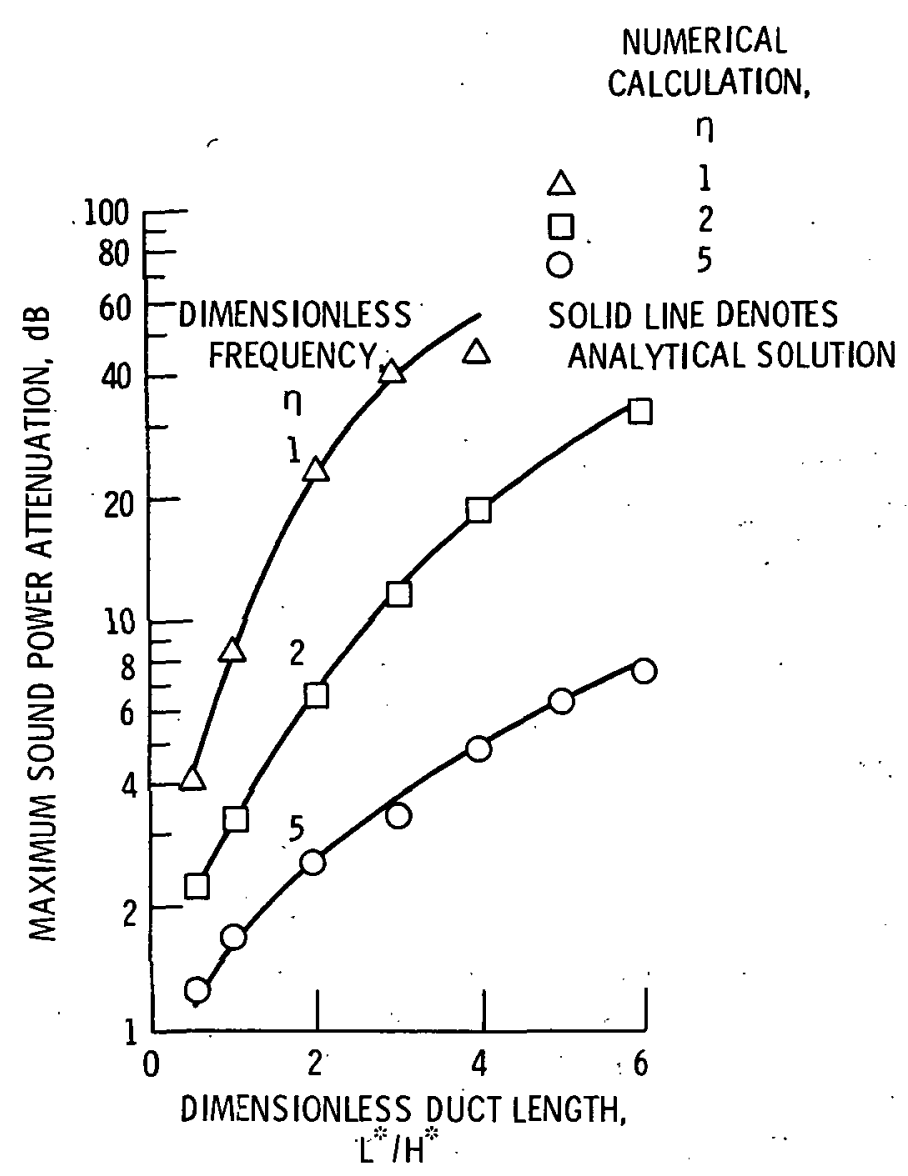

Figure 5. - Effect of axial length and frequency on attenuation at optimum impedance in two-dimensional duct for a plane wave input. $(I=31$, $\mathrm{J}=20, \mathrm{t}=5.0, \Delta \mathrm{t}=0.5 \mathrm{x} \Delta \mathrm{t}_{\max }$ ). 


\begin{tabular}{|c|c|}
\hline $\begin{array}{l}\text { 1. Report No. } \\
\text { NASA TM-83744 }\end{array}$ & 3. Recipient's Catalog No. \\
\hline $\begin{array}{l}\text { 4. Title and Subtitle } \\
\text { Time Dependent Wave Envelope Finite Difference } \\
\text { Analys is of Sound Propagation. }\end{array}$ & $\begin{array}{l}\text { 6. Performing Organization Code } \\
505-31-3 B\end{array}$ \\
\hline \multirow{2}{*}{ Kenneth J. Baume ister } & $\begin{array}{l}\text { 8. Performing Organization Report No. } \\
\text { E-2233 }\end{array}$ \\
\hline & 10. Work Unit No. \\
\hline \multirow{2}{*}{$\begin{array}{l}\text { 9. Performing Organization Name and Address } \\
\text { National Aeronautics and Space Administration } \\
\text { Lewis Research Center } \\
\text { Cleveland, Ohio } 44135\end{array}$} & \\
\hline & 13. Type of Report and Period Covered \\
\hline \multirow{2}{*}{$\begin{array}{l}\text { 12. Sponsoring Agency Name and Address } \\
\text { National Aeronautics and Space Administration } \\
\text { Washington, D.C. } 20546\end{array}$} & Technical Memorandum \\
\hline & 14. Sponsoring Agency Code \\
\hline \multicolumn{2}{|c|}{$\begin{array}{l}\text { 15. Supplementary Notes } \\
\text { Prepared for the Ninth Aeroacoustics Conference sponsored by the American } \\
\text { Institue of Aeronautics and Astronautics, Williamsburg, Virginia, October 15-17, } \\
1984 .\end{array}$} \\
\hline \multicolumn{2}{|c|}{$\begin{array}{l}\text { Abstract } \\
\text { A transient finite-difference wave envelope formulation is presented for sound } \\
\text { mulated, the governing wave equation is first transformed to a form whose solution } \\
\text { tends not to oscillate along the propagation direction. This transformation re- } \\
\text { duces the required number of grid points by an order of magnitude. Physically, } \\
\text { the transformed pressure represents the amplitude of the conventional sound wave. } \\
\text { The derivation for the wave envelope transient wave equation and appropriate } \\
\text { boundary conditions are presented as well as the difference equations and stabil- } \\
\text { ity requirements. To illustrate the method, example solutions are presented for } \\
\text { sound propagation in a straight hard-wall duct and in a two-dimensional straight } \\
\text { soft-wall duct. The numerical results are in good agreement with exact analytical } \\
\text { results. }\end{array}$} \\
\hline
\end{tabular}

17. Key Words (Suggested by Author(s))

Finite difference; Transient; Waveenvelope; Acoustics; Duct propagation 18. Distribution Statement
Unclassified - un 1 imited
STAR Category 71

19. Security Classif. (of this report) Unclassified 20. Security Classif. (of this page)
Unclass ified 21. No. of pages 22. Price 
National Aeronautics and Space Administration

Washington, D.C.

20546

Official Business

Penalty for Private Use, $\mathbf{5 3 0 0}$
SPECIAL FOUATH CLASS MAIL BOOK

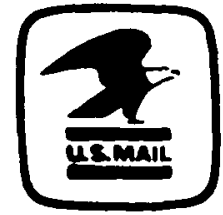

Postege and Fees Paid National Aeronautics and Space Administration NASA-451 